\title{
Type 1 Gall Bladder Perforation As A Rare Complication of Isolated Choledocholithiasis
}

\author{
RanendraHajong, K Lenish Singh, DebobrattaHajong, NarangNaku, \\ MadhurAnand, NilanjanMajumdar \\ Department of Surgery, NEIGRIHMS, Shillong
}

\begin{abstract}
:
Background: Perforation of the gall bladder due to choledocholithiasis is a rare cause of peritonitis. We present a case of gallbladder perforation and biliary peritonitis due to choledocholithiasis.

Case report: A 50 years old male, without any known medical co-morbidity, presented with a 5-day history abdominal pain and distension. On abdominal examination, distension with rebound tenderness was present. MRI showed a gallbladder perforation. The patient was taken up for an emergency exploratory laparotomy which showed biliary peritonitis, perforation of gallbladder and choledocholithiasis. Cholecystectomyand choledocholithotomy done with T-tube placement. The patient's hospital course was uneventful except for the initial part when he required ventilator support and dialysis.

Conclusion: Gallbladder perforation is an unusual initial presentation of gallbladder disease. Earlydiagnosis of gallbladder perforation and immediate surgical intervention are of prime importancein decreasing morbidity and mortality associated with this condition.
\end{abstract}

Keywords: Gallbladder perforation; type I; choledocholithiasis; isolated.

\section{Introduction}

Perforation of gallbladder is a life threatening complication of acute cholecystitis with increased morbidity and mortality [1]The etiopathogenesisof gallbladder perforation is poorly understood and diagnostic as well as therapeutic dilemmas are commonly faced by the treating surgeons [2]. We present a rare case of gallbladder perforation and biliary peritonitis due to impacted choledocholithiasisin a 50 years old male which caused diagnostic dilemma at presentation but was managed successfully.

\section{Case report:}

A 50 years old gentleman presented to NEIGRIHMS hospital with complaints of pain in the upper abdomen, fever, rigors and vomiting for 5 days with inability to pass flatus. He did not have any other illness or addiction. The patient at admission was drowsy, icteric\& dehydrated with tachycardia, tachypnea and blood pressure of $88 / 52 \mathrm{mmHg}$. Abdomen was distended and tender all over with rebound tenderness, intact liver dullness and absent bowel sounds. The patient was resuscitated and a bedside ultrasonography did not reveal any significant finding. Liver enzymes and bilirubin were elevated.Serum creatinine was raised by 6 times the normal level. A non-contrast MRI (magnetic resonance imaging) was done which showed grossly distended edematous gallbladder (GB),pericholecystic collection and pneumobilia. HASTE Transverse Fat sat image revealed a defect in the GB wall along the GB fossa with extravasating fluid into the GB fossa (Figure 1). As the patient's creatinine was very high, the patient was put on hemodialysis and after four sittings of hemodialysis, the patient was taken up forlaparotomy when the creatinine level came down. At operation gall-bladder perforation was detected in the body region (Figure 2). No stone was detected in the gallbladder but a1 $\mathrm{cm}$ stone was palpated in the distal common bile duct was removed by a choledochotomy and bile duct closedover a Ttube. Postoperatively the patient recovered uneventfully.

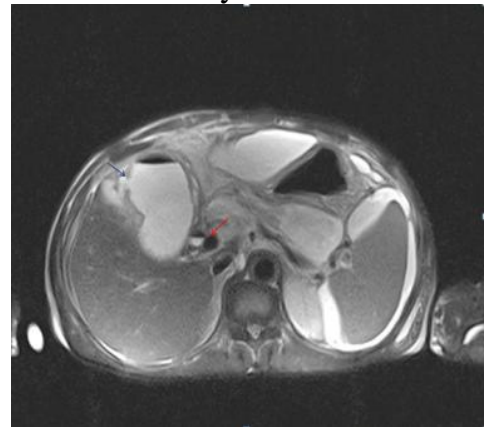

Figure 1 showing gallbladder perforation in the body (Blue arrow) and air in the common bile duct (Red arrow) 


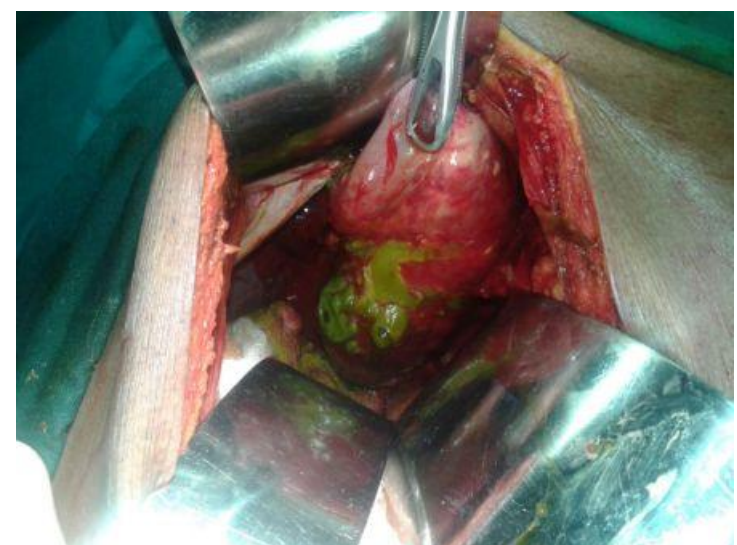

Figure 2 showing bile leaking from perforated gallbladder

\section{Discussion}

Gallbladder perforations may be caused by traumatic, iatrogenic or idiopathic aetiologies and carry a high mortality rate of about $12-16 \%$. Cholelithiasis, infections, malignancy, steroid therapy, diabetes mellitus and atherosclerotic heart disease may predispose a patient for gallbladder perforation [3]. Niemeier classified gallbladder perforation into three types as: a) Type I or acute perforation presenting with generalized peritonitis; b) Type II or subacute with pericholecystic abscess and localized peritonitis; and c) Type III or chronic presenting with cholecystoenteric fistula [4]. Ourpatient had a type 1 gallbladder perforation.

Ultrasonography is first investigation done in most of the cases, and may show distended gallbladder $(>3.5-4 \mathrm{~cm})$ with thick wall $(>3 \mathrm{~mm})$,pericholecystic fluid collection and 'hole sign'which reliably and specifically indicates gall bladder perforation [1].

Computed tomography (CT) scan is the most sensitive test for detection of gall bladder perforation. Stones outside the lumen of gallbladder or a ruptured part of the gall bladder wall are direct indicators of perforated gallbladder whereas abscess outside the gall bladder is indirect indicator [1].

Tonolini et al recommend using MRI for early and efficient triage of patients with suspected cholecystitis with equivocal clinical, laboratory, and sonographic findingsbecause of its excellent ability to detect stones in the biliary tree, biliary dilatation, and the relationship of a pericholecystic fluid collection to the abdominal wall and gallbladder which helps in surgical planning [5,6].

Open or laparoscopic cholecystectomy with drainage of the collections and any associated liver abscesses and thorough peritoneal lavage is the treatment of choice. However in type III gallbladder perforation, the cholecysto-enteric fistula needs to be repaired [1].

\section{Conclusion}

Gallbladder perforation is a rare but life threatening emergency situation which frequently poses diagnostic dilemma. In the available literature we could not find any case of gallbladder perforation due to common bile duct stones without cholelithiasis, which indicates that it is a rare entity. High index of suspicion and aggressive approach for workup and surgery is imperative for diagnosis and treatment.

\section{Conflicts of interest: None}

\section{References}

[1]. Jethwani U, Singh G, Mohil RS, Saroha R, Chouhan J, BansalN. Gall bladder perforation: report of two cases. OA Case Reports 2013;2(5):50.

[2]. Date RS, Thrumurthy SG, Whiteside S, Umer MA, Pursnani KG, Ward JB et al. Gallbladder perforation: Case series and systematic review. Int J Surg 2012;10(2):63-68.

[3]. Stefanidis D, Sirinek KR, Bingener J. Gallbladder Perforation: Risk Factors and Outcome. J Surg Res 2006;131(2):204-8.

[4]. Alvi AR, Ajmal S, Saleem T. Acute free perforation of gall bladder encountered at initial presentation in a 51 years old man: a case report. Cases J 2009;2(1):166.

[5]. TonoliniM, Ravelli A, Villa C, Bianco R.Urgent MRI with MR cholangiopancreatography (MRCP) of acute cholecystitis and related complications: diagnostic role and spectrum of imaging findings. EmergRadiol 2012;19(4):341-8.

[6]. Karcaaltincaba M, Hohenwalter MD, Erickson SJ, Taylor AJ. MRCP findings of gallbladder perforation and pericholecystic abscess. CMIG Extra: Cases. 2004;28:59-61. 